Annals of Pure and Applied Mathematics

Vol. 18, No. 2, 2018, 201-206

ISSN: 2279-087X (P), 2279-0888(online)

Published on 28 December 2018

www.researchmathsci.org

DOI: http://dx.doi.org/10.22457/apam.v18n2a10

Annals of

Pure and Applied

Mathematics

\title{
Degree Based Connectivity $F$-Indices of Nanotubes
}

\author{
V.R.Kulli
}

Department of Mathematics

Gulbarga University, Gulbarga 585 106, India

E-mail: vrkulli@gmail.com

Received 25 November 2018; accepted 27 December 2018

Abstract. The connectivity indices are helpful for medical scientists, chemical scientists to find out the chemical and biological characteristics of drugs. In this study, we compute the sum connectivity $F$-index, product connectivity $F$-index, atom bond connectivity $F$ index and geometric arithmetic $F$-index of certain nanotubes.

Keywords: connectivity $F$-indices, nanotube

AMS Mathematics Subject Classification (2010): 05C05, 05C07, $05 C 35$

\section{Introduction}

We consider only finite, simple connected graphs. Let $G$ be a connected graph with vertex set $V(G)$ and edge set $E(G)$. The degree $d_{G}(v)$ of a vertex $v$ is the number of edges incident to $v$. We refer to [1] for undefined term and notation. A topological index is a numerical parameter mathematically derived from the graph structure. The connectivity indices are used in the analysis of drug molecular structures in Chemical and Medical Sciences. Several topological indices have been considered in Chemistry and have found some applications, especially in QSPR/QSAR study, see [2, 3].

The first $F$-index [4] and second $F$-index [5] of a graph $G$ are defined as

$$
F_{1}(G)=\sum_{u v E(G)}\left[d_{G}(u)^{2}+d_{G}(v)^{2}\right], \quad F_{2}(G)=\sum_{u v \in E(G)}\left[d_{G}(u)^{2} d_{G}(v)^{2}\right] .
$$

The sum connectivity $F$-index and product connectivity $F$-index were introduced by Kulli in [5] and defined as

$$
\begin{aligned}
& S F(G)=\sum_{u v \in E(G)} \frac{1}{\sqrt{d_{G}(u)^{2}+d_{G}(v)^{2}}} \\
& P F(G)=\sum_{u v \in E(G)} \frac{1}{\sqrt{d_{G}(u)^{2} d_{G}(v)^{2}}}
\end{aligned}
$$

We now introduce the atom bond connectivity $F$-index and geometric arithmetic $F$-index of a graph $G$, defined as

$$
A B C F(G)=\sum_{u v \in E(G)} \sqrt{\frac{d_{G}(u)^{2}+d_{G}(v)^{2}-2}{d_{G}(u)^{2} d_{G}(v)^{2}}}
$$




$$
P F(G)=\sum_{u v \in E(G)} \frac{2 \sqrt{d_{G}(u)^{2} d_{G}(v)^{2}}}{d_{G}(u)^{2}+d_{G}(v)^{2}}
$$

Recently, the reduced connectivity indices [6], product connectivity leap index and $\mathrm{ABC}$ leap index [7], atom bond connectivity index [9], multiplicative connectivity ve-degree indices [10], multiplicative connectivity Banhatti indices [11], multiplicative connectivity Revan indices [12], sum connectivity leap index [13], multiplicative atom bond connectivity index [14] were studied. In this paper, the sum connectivity $F$-Index, product connectivity $F$-index, atom bond connectivity $F$-Index and geometric arithmetic $F$-index of $\mathrm{HC}_{5} C_{7}[p, q]$ and $S C_{5} C_{7}[p, q]$ nanotubes are determined. For nanotubes, see [15].

\section{2. $H C_{5} C_{7}[p, q]$ Nanotubes}

We consider nanotubes, denoted by $\mathrm{HC}_{5} C_{7}[p, q]$, in which $p$ is the number of heptagons in the first row and $q$ rows of pentagons repeated alternately. The 2-D lattice of nanotube $\mathrm{HC}_{5} \mathrm{C}_{7}[p, q]$ is depicted in Figure 1.

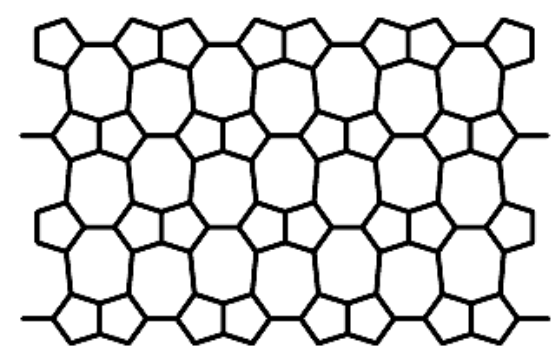

Figure 1: 2-dimensional lattice of nanotube $\mathrm{HC}_{5} \mathrm{C}_{7}[8,4]$

Let $G$ be the graph of a nanotube $H C_{5} C_{7}[p, q]$. By algebraic method, we obtain that $G$ has $4 p q$ vertices and $6 p q-p$ edges. The graph $G$ has two types of edges based on the degree of end vertices of each edge as given in Table 1.

\begin{tabular}{lcc}
\hline $\mathrm{d}_{G}(u), d_{G}(v) \backslash u v \in E(G)$ & $(2,3)$ & $(3,3)$ \\
\hline Number of edges & $4 p$ & $6 p q-5 p$ \\
\hline
\end{tabular}

Table 1: Edge partition of $H_{5} C_{7}[p, q]$

Theorem 1. The sum connectivity $F$-index of a nanotube $H C_{5} C_{7}[p, q]$ is

$$
S F\left(H C_{5} C_{7}[p, q]\right)=\sqrt{2} p q+\left(\frac{4}{\sqrt{13}}-\frac{5}{\sqrt{18}}\right) p
$$

Proof: Let $G=H C_{5} C_{7}[p, q]$. By using equation (1) and Table 1, we derive

$$
\begin{aligned}
& S F\left(H C_{5} C_{7}[p, q]\right)=\sum_{u v \in E(G)} \frac{1}{\sqrt{d_{G}(u)^{2}+d_{G}(v)^{2}}} \\
& =\left(\frac{1}{\sqrt{2^{2}+3^{2}}}\right) 4 p+\left(\frac{1}{\sqrt{3^{2}+3^{2}}}\right)(6 p q-5 p)
\end{aligned}
$$


Degree Based Connectivity $F$-Indices of Nanotubes

$$
=\sqrt{2} p q+\left(\frac{4}{\sqrt{13}}-\frac{5}{\sqrt{18}}\right) p \text {. }
$$

Theorem 2. The product connectivity $F$-index of a nanotube $H C_{5} C_{7}[p, q]$ is given by

$$
P F\left(H C_{5} C_{7}[p, q]\right)=\frac{2}{3} p q+\frac{1}{9} p .
$$

Proof: Let $G=H C_{5} C_{7}[p, q]$. From equation (2) and by using Table 1, we deduce

$$
\begin{aligned}
& P F\left(H C_{5} C_{7}[p, q]\right)=\sum_{u v \in E(G)} \frac{1}{\sqrt{d_{G}(u)^{2} d_{G}(v)^{2}}} \\
= & \left(\frac{1}{\sqrt{2^{2} \times 3^{2}}}\right) 4 p+\left(\frac{1}{\sqrt{3^{2} \times 3^{2}}}\right)(6 p q-5 p) \\
= & \frac{2}{3} p q+\frac{1}{9} p .
\end{aligned}
$$

Theorem 3. The atom bond connectivity $F$-index of a nanotube $H C_{5} C_{7}[p, q]$ is

$$
A B C F\left(H C_{5} C_{7}[p, q]\right)=\frac{8}{3} p q+\left(\frac{2 \sqrt{11}}{3}-\frac{20}{9}\right) p
$$

Proof: Let $G=H C_{5} C_{7}[p, q]$. By using equation (3) and Table 1, we obtain

$$
\begin{aligned}
& A B C F\left(H C_{5} C_{7}[p, q]\right)=\sum_{u v \in E(G)} \sqrt{\frac{d_{G}(u)^{2}+d_{G}(v)^{2}-2}{d_{G}(u)^{2} d_{G}(v)^{2}}} \\
& =\left(\sqrt{\frac{2^{2}+3^{2}-2}{2^{2} \times 3^{2}}}\right) 4 p+\left(\sqrt{\frac{3^{2}+3^{2}-2}{3^{2} \times 3^{2}}}\right)(6 p q-5 p) \\
& =\frac{8}{3} p q+\left(\frac{2 \sqrt{11}}{3}-\frac{20}{9}\right) p .
\end{aligned}
$$

Theorem 4. The geometric-arithmetic $F$-index of a nanotube $H_{5} C_{7}[p, q]$ is given by

$$
G A F\left(H C_{5} C_{7}[p, q]\right)=6 p q-\frac{17}{13} p .
$$

Proof: Let $G$ be the graph of a nanotube $H C_{5} C_{7}[p, q]$. From equation (4) and by using Table 1, we have

$$
\begin{aligned}
& G A F\left(H C_{5} C_{7}[p, q]\right)=\sum_{u v \in E(G)} \frac{2 \sqrt{d_{G}(u)^{2} d_{G}(v)^{2}}}{d_{G}(u)^{2}+d_{G}(v)^{2}} \\
& =\left(\frac{2 \sqrt{2^{2} \times 3^{2}}}{2^{2}+3^{2}}\right) 4 p+\left(\frac{2 \sqrt{3^{2} \times 3^{2}}}{3^{2}+3^{2}}\right)(6 p q-5 p) \\
& =6 p q-\frac{17}{13} p .
\end{aligned}
$$




\section{3. $S C_{5} C_{7}[p, q]$ Nanotubes}

We consider $S C_{5} C_{7}[p, q]$ nanotubes in which $p$ is the number of heptagons in the first row and $q$ rows of vertices and edges are repeated alternately. The 2- $D$ lattice a nanotube $S C_{5} C_{7}[p, q]$ is presented in Figure 2.

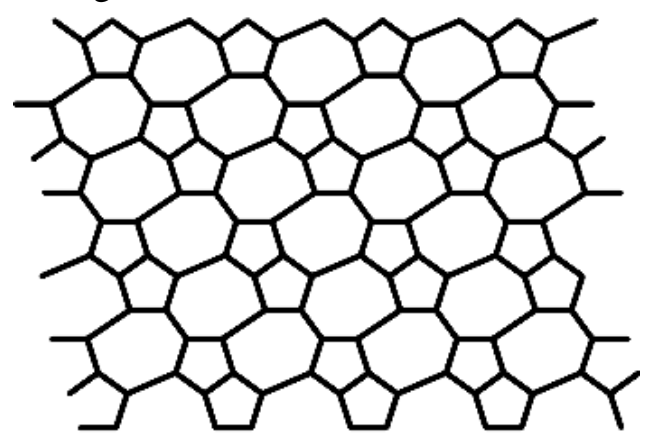

Figure 2: 2-dimensional lattice of nanotube $\mathrm{SC}_{5} \mathrm{C}_{7}[p, q]$

Let $G$ be the graph of a nanotube $S C_{5} C_{7}[p, q]$ with $6 p q-p$ edges. By algebraic method, we obtain that $G$ has three types of edges based on the degree of the end vertices of each edge as given in Table 2.

\begin{tabular}{lccc}
\hline$d_{G}(u) d_{G}(v) \backslash u v \in E(G)$ & $(2,2)$ & $(2,3)$ & $(3.3)$ \\
\hline Number of edges & $q$ & $6 q$ & $6 p q-p-7 q$ \\
\hline
\end{tabular}

Table 2: Edge partition of $\mathrm{SC}_{5} \mathrm{C}_{7}[p q]$

Theorem 5. The sum connectivity $F$-index of a nanotube $S C_{5} C_{7}[p, q]$ is given by

$$
S F\left(S C_{5} C_{7}[p, q]\right)=\sqrt{2} p q-\frac{1}{\sqrt{18}} p+\left(\frac{1}{\sqrt{8}}+\frac{6}{\sqrt{13}}-\frac{7}{\sqrt{18}}\right) q .
$$

Proof: Let $G$ be the graph of a nanotube $S C_{5} C_{7}[p, q]$. From equation (1) and by using Table 2, we obtain

$$
\begin{aligned}
& S F\left(S C_{5} C_{7}[p, q]\right)=\sum_{u v E(G)} \frac{1}{\sqrt{d_{G}(u)^{2}+d_{G}(v)^{2}}} \\
& =\left(\frac{1}{\sqrt{2^{2}+2^{2}}}\right) q+\left(\frac{1}{\sqrt{2^{2}+3^{2}}}\right) 6 q+\left(\frac{1}{\sqrt{3^{2}+3^{2}}}\right)(6 p q-p-7 q) \\
& =\sqrt{2} p q-\frac{1}{\sqrt{18}} p+\left(\frac{1}{\sqrt{8}}+\frac{6}{\sqrt{13}}-\frac{7}{\sqrt{18}}\right) q .
\end{aligned}
$$

Theorem 6. The product connectivity $F$-index of $S C_{5} C_{7}[p, q]$ is

$$
P F\left(S C_{5} C_{7}[p, q]\right)=\frac{2}{3} p q-\frac{1}{9} p+\frac{17}{36} q .
$$

Proof: Let $G=S C_{5} C_{7}[p, q]$. By using equation (2) and Table 2, we derive

$$
P F\left(S C_{5} C_{7}[p, q]\right)=\sum_{u v \in E(G)} \frac{1}{\sqrt{d_{G}(u)^{2} d_{G}(v)^{2}}}
$$


Degree Based Connectivity $F$-Indices of Nanotubes

$$
\begin{aligned}
& =\left(\frac{1}{\sqrt{2^{2} \times 2^{2}}}\right) q+\left(\frac{1}{\sqrt{2^{2} \times 3^{2}}}\right) 6 q+\left(\frac{1}{\sqrt{3^{2} \times 3^{2}}}\right)(6 p q-p-5 q) \\
& =\frac{2}{3} p q-\frac{1}{9} p+\frac{17}{36} q .
\end{aligned}
$$

Theorem 7. The atom bond connectivity $F$-index of $S C_{5} C_{7}[p, q]$ is

$$
A B C F\left(S C_{5} C_{7}[p, q]\right)=\frac{8}{3} p q-\frac{4}{9} p+\left(\frac{\sqrt{6}}{4}+\sqrt{11}-\frac{28}{9}\right) q .
$$

Proof: Let $G$ be the graph of a nanotube $S C_{5} C_{7}[p, q]$. By using equation (3) and Table 2, we obtain

$$
\begin{aligned}
& A B C F\left(S C_{5} C_{7}[p, q]\right)=\sum_{u v \in E(G)} \sqrt{\frac{d_{G}(u)^{2}+d_{G}(v)^{2}-2}{d_{G}(u)^{2} d_{G}(v)^{2}}} \\
& =\left(\sqrt{\frac{2^{2}+2^{2}-2}{2^{2} \times 2^{2}}}\right) q+\left(\sqrt{\frac{2^{2}+3^{2}-2}{2^{2} \times 3^{2}}}\right) 6 q+\left(\sqrt{\frac{3^{2}+3^{2}-2}{3^{2} \times 3^{2}}}\right)(6 p q-p-7 q) \\
& =\frac{8}{3} p q-\frac{4}{9} p+\left(\frac{\sqrt{6}}{4}+\sqrt{11}-\frac{28}{9}\right) q .
\end{aligned}
$$

Theorem 8. The geometric-arithmetic $F$-index of $S C_{5} C_{7}[p, q]$ is given by

$$
G A F\left(S C_{5} C_{7}[p, q]\right)=6 p q-p-\frac{66}{13} q .
$$

Proof: Let $G=S C_{5} C_{7}[p, q]$. From equation (4) and by using Table 2, we have

$$
\begin{aligned}
& G A F\left(S C_{5} C_{7}[p, q]\right)=\sum_{u v \in E(G)} \frac{2 \sqrt{d_{G}(u)^{2} d_{G}(v)^{2}}}{d_{G}(u)^{2}+d_{G}(v)^{2}} \\
& =\left(\frac{2 \sqrt{2^{2} \times 2^{2}}}{2^{2}+2^{2}}\right) p+\left(\frac{2 \sqrt{2^{2} \times 3^{2}}}{2^{2}+3^{2}}\right) 6 q+\left(\frac{2 \sqrt{3^{2} \times 3^{2}}}{3^{2}+3^{2}}\right)(6 p q-p-7 q) \\
& =6 p q-p-\frac{66}{13} q .
\end{aligned}
$$

Acknowledgement. The author is thankful to the referee for useful comments.

\section{REFERENCES}

1. V.R.Kulli, College Graph Theory, Vishwa International Publications, Gulbarga, India (2012).

2. I.Gutman and O.E.Polansky, Mathematical Concepts in Organic Chemistry, Springer, Berlin (1986).

3. V.R.Kulli, Multiplicative Connectivity Indices of Nanostructures, LAP LEMBERT Academic Publishing, (2018).

4. B.Furtula and I.Gutman, A forgotten topological index, J. Math. Chem. 53 (2015), 1184-1190.

5. V.R.Kulli, $F$-indices of chemical networks, submitted. 
6. V.R.Kulli, Computing reduced connectivity indices of certain nanotubes, Journal of Chemistry and Chemical Sciences, 8(11) (2018) 1174-1180.

7. V.R.Kulli, Product connectivity leap index and $\mathrm{ABC}$ leap index of helm graphs, Annals of Pure and Applied Mathematics, 18(2) (2018) 189-193.

8. V.R.Kulli, Atom bond connectivity reverse and product connectivity reverse indices of oxide and honeycomb networks, International Journal of Fuzzy Mathematical Archive, 15(1) (2018) 1-5.

9. V.R.Kulli, The sum connectivity Revan index of silicate and hexagonal networks, Annals of Pure and Applied Mathematics, 14(3) (2017) 401-406.

10. V.R.Kulli, Multiplicative connectivity ve-degree indices of dominating oxide and regular triangulate oxide networks, International Journal of Current Advanced Research, 7, 4(J) (2018) 11961-11964.

11. V.R.Kulli, Multiplicative connectivity Banhatti indices of dendrimer nanostars, Journal of Chemistry and Chemical Sciences, 8(6) (2018) 964-973.

12. V.R.Kulli, Multiplicative connectivity Revan indices of polycyclic aromatic hydrocarbons and benzenoid systems, Annals of Pure and Applied Mathematics, 16(2) (2018) 337-343.

13. V.R.Kulli, Sum connectivity leap index and geometric-arithmetic leap index of certain windmill graphs, Journal of Global Research in Mathematical Archives, 6(1) (2019).

14. V.R.Kulli and M.H.Akhabari, Multiplicative atom bond connectivity and multiplicative geometric-arithmetic indices of dendrimer nanostars, Annals of Pure and Applied Mathematics, 16(2) (2018) 429-436.

15. I.Iranmanesh and M.Zeraatkar, Computing GA index for nanotubes, Optoelectron. Adv. Mater. Rapid Commun. 4(11) (2010) 1852-1855. 